\title{
Hot topics from the Assemblies
}

Cite as: Hot topics from the Assemblies. Breathe 2018; 14: e118.

\section{Nocturnal oximetry-based evaluation of habitually snoring children}

Authors: Hornero R, Kheirandish-Gozal L, GutiérrezTobal GC, et al.

Am J Respir Crit Care Med 2017; 196: 1591-1598. Summary: Obstructive sleep apnoea (OSA) is prevalent and polysomnography (PSG) is the gold standard in diagnosis. However, PSG is not always routinely available and many children are treated empirically. Nocturnal oximetry is emerging as a widely available, simple and cost-effective screening alternative with the main limitation being low interobserver reliability and relatively low accuracy.

The aim of this study was to assess the diagnostic accuracy of a new automated neural network algorithm, using the pulse oximetry signal in snoring children aged $2-18$ years. The oximetry signal was extracted from formal nocturnal PSG. 4191 children were analysed in 13 sleep laboratories around the world. Overall, the accuracy ranged between 75\% and $90 \%$ for apnoea-hypopnoea index (AHI) of 1-10 events $\cdot h^{-1}$, with higher sensitivity at lower $\mathrm{AHI}$ and higher specificity at higher $\mathrm{AHI}$. Therefore, $\mathrm{AHI}<1$ events $\cdot \mathrm{h}^{-1}$ would exclude OSA $(94.4 \%$ would have PSG $\mathrm{AHI}<5$ events $\left.\cdot \mathrm{h}^{-1}\right) ; \mathrm{AHI} \geq 5$ events $\cdot \mathrm{h}^{-1}$ would be referred for treatment $(94.4 \%$ would have a PSG $A H I \geq 1$ events $\left.\cdot h^{-1}\right) ; A H I \geq 1$ and $<5$ events $\cdot h^{-1}$ would be referred for PSG. This protocol would reduce the overall need for PSG by $52.6 \%$.

This study confirms the accuracy of nocturnal oximetry, using a specific algorithm beyond conventional indexes, as a screening tool for paediatric OSA in snoring children. The results are important because this screening algorithm would enable better selection of those children who would benefit from a PSG and would reduce the number of children being treated empirically, reducing costs and unnecessary procedures.

Reviewed by: Andrés F. Almario-Hernández, Maria Cols-Roig (Spain, Assembly 7)
The association between Staphylococcus aureus and subsequent bronchiectasis in children with cystic fibrosis

Authors: Caudri D, Turkovic L, Ng J, et al.

J Cyst Fibros 2018; 17: 462-469.

Summary: Staphyloccocus aureus is a bacterial pathogen commonly isolated from the airways of cystic fibrosis (CF) patients. The impact of its presence on the development and later progression of lung disease is lacking. The AREST (Australian Respiratory Early Surveillance Team) longitudinal birth cohort study collected data from 2005 to 2016 in two hospitals in Australia. Patients were recruited from 3 months of age and received annual bronchoscopy with bronchoalveolar lavage sent for culture and a chest computed tomography (CT) scan. From the age of 4 years onwards spirometry was performed on a 3-monthly basis. A total of 368 children were recruited; 219 children remained in the study until the age of 5 years.

S. aureus was isolated from $9.8 \%$ of subjects at 3 years of age. This was significantly associated with a subsequent increase in bronchiectasis score and lower forced expiratory flow at $25-75 \%$ of forced vital capacity, but not forced expiratory volume in $1 \mathrm{~s}$ (FEV1) \% predicted.

This study had a large sample size with prospective follow-up. It could partially explain why previous studies could not demonstrate any beneficial effect of prophylaxis against $S$. aureus as they relied on measurement of FEV1.

Further studies are needed to demonstrate the effect of prophylaxis or eradication regimens for S. aureus using sophisticated pulmonary function test measurements and early $\mathrm{CT}$ scans to detect structural changes.

Reviewed by: Muna Mokbel Dahabreh Jordan, Assembly 7) 